PRACTICE CMAJ

\title{
Cutaneous sarcoidosis at insulin injection sites
}

\section{Hanieh Zargham, Elizabeth O'Brien MD}

Competing interests: None declared.

This article has been peer reviewed.

The authors have obtained patient consent.

Affiliations: Departments of Medicine (Zargham) and Dermatology (O'Brien), McGill University Health Centre, Montréal, Que.

Acknowledgements: The authors thank Dr. Ivan Litvinov, McGill University Department of Dermatology, and Dr. A.K. Watters, McGill University Department of Pathology, for their assistance with obtaining and interpreting the pathology results.

\section{Correspondence to:} Hanieh Zargham, hanieh .zargham@mail.mcgill.ca

CMAJ 2016. DOI:10.1503 /cmaj.150572
A 49-year-old woman with diabetes presented with a three-month history of asymptomatic skin lesions on her abdomen at the sites of insulin injection (Figure 1), as well as in an old scar on her knee. She recalled similar lesions at sites of minor trauma and in another scar eight years earlier. In the past, the lesions had not been biopsied and had resolved with intralesional injection of corticosteroids.

Based on the bilateral symmetric distribution and the location of the abdominal lesions at insulin injection sites, our differential diagnosis included a foreign-body reaction and traumatic induction (koebnerization) of sarcoidosis, granuloma annulare and lichen planus. The brownish-red colour of the lesions was clinically more compatible with sarcoidosis. Granuloma annulare and lichen planus tend to have a violet hue. In addition, sarcoidosis can occur in old, previously stable scars. ${ }^{1}$

A biopsy of the patient's skin was consistent with sarcoidosis (Figure 2). Investigations including complete blood count, serum calcium level, serum angiotensin-converting enzyme level and chest radiography showed normal results. We prescribed clobetasol proprionate cream to be applied topically, and the lesions decreased in size and colour intensity after two months.

A diagnosis of sarcoidosis requires a compatible clinical picture, the demonstration of noncaseating granulomas on tissue biopsy and the exclusion of other processes with similar clinical and histopathologic presentations. Although the lung is the most frequently involved organ, about $30 \%$ of patients present with extrapulmonary lesions, with cutaneous sarcoidosis affecting up to $25 \%$ of all patients. ${ }^{2}$ Cutaneous involvement may be the first and only manifestation of sarcoidosis, but all patients with cutaneous sarcoidosis should be evaluated and followed up for systemic disease. ${ }^{3}$ Cutaneous sarcoidosis typically presents as red-brown papules and plaques distributed symmetrically on the face, lips, neck, upper trunk and extremities. ${ }^{3}$

Granulomas commonly appear in scar tissue ${ }^{1}$ and can also be found at sites of skin trauma, which may represent koebnerization. In our patient's case, trauma from the subcutaneous injection of insulin may have introduced foreign

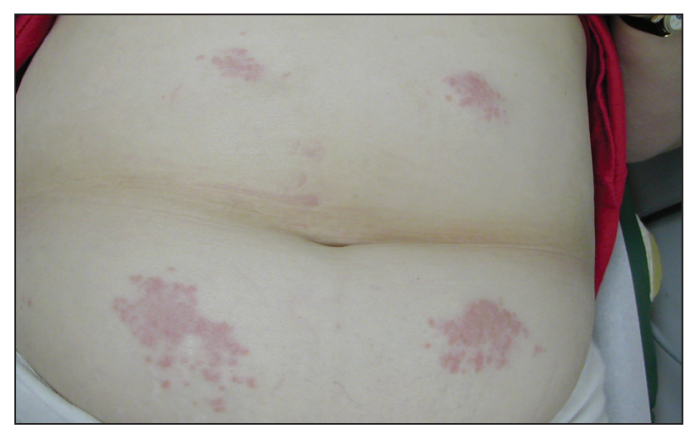

Figure 1: Confluent brownish-red papules and plaques distributed symmetrically at the abdominal insulin injection sites of a 49-year-old woman with diabetes.

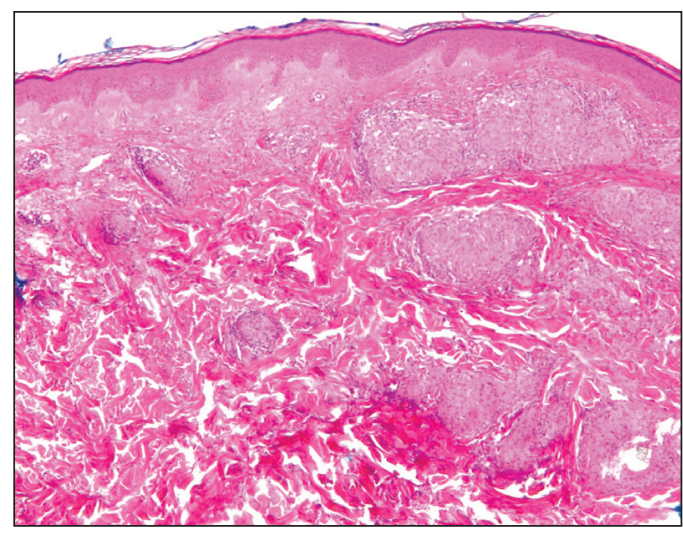

Figure 2: Histologic specimen from a lesion on the patient's abdomen, showing noncaseating, microorganism-negative granulomas with focal foreign-body giant cell reaction to nonpolarizable foreign material. Hematoxylin and eosin stain. Original magnification $\times \mathbf{2 . 5}$.

particles that acted as a nidus for granuloma formation. ${ }^{1}$ The recommended workup for sarcoidosis includes a complete physical examination, chest radiography, pulmonary function testing, electrocardiography, tuberculin skin testing, urinalysis, complete blood count and serum levels of calcium and angiotensin-converting enzyme. ${ }^{3}$

\section{References}

1. Marcoval J, Fanlo M, Penín R, et al. Systemic sarcoidosis with specific cutaneous lesions located at insulin injection sites for diabetes mellitus. J Eur Acad Dermatol Venereol 2014;28:1259-60.

2. Iannuzzi MC, Rybicki B, Teirstein A. Sarcoidosis. $N$ Engl $J$ Med 2007;357:2153-65.

3. Wanat KA, Rosenbach M. A Practical approach to cutaneous sarcoidosis. Am J Clin Dermatol 2014;15:283-97. 